\title{
An Evaluation of Individually Delivered Secret Agent Society Social Skills Program for Children with High-Functioning Autism Spectrum Disorders: A pilot study
}

\author{
Yunxi Lynette Tan \\ Curtin University \\ Trevor G. Mazzucchelli \\ Curtin University and The University of Queensland \\ Renae Beaumont \\ The University of Queensland
}

Author Note

Yunxi Lynette Tan, Brain, Behaviour and Mental Health Research Group, School of Psychology and Speech Pathology, Curtin University. Trevor G. Mazzucchelli, Brain, Behaviour and Mental Health Research Group, School of Psychology and Speech Pathology, Curtin University and Parenting and Family Support Centre, School of Psychology, The University of Queensland; Renae Beaumont, School of Psychology, The University of Queensland.

This research was supported in part by a grant awarded to Trevor Mazzucchelli and Lynette Tan from the School of Psychology and Speech Pathology Research Allocation Fund RAF-2013-03. Practitioner and parent resources, as well as practitioner training were provided by the Social Skills Training Institute, a division of Triple P International Pty Ltd.

We thank Amy Hamilton, Yeow May Tan and Nick Buckley for assisting in scoring the test protocols, as well as Theresa Kidd and Josette Hamilton for helping with the recruitment of participants.

Conflict of interest statement. The third author of this paper, Dr Renae Beaumont, is the developer of the Secret Agent Society social-emotional skills program, and receives royalties on all program materials sold.

Correspondence concerning this article should be addressed to Trevor G. Mazzucchelli, School of Psychology and Speech Pathology, Curtin University, Perth, Western Australia, Australia. E-mail: trevor.mazzucchelli@curtin.edu.au. Facsimile: +8 9266 2464. Telephone: +892667182 . 


\begin{abstract}
High-functioning children with Autism Spectrum Disorders display social skill deficits that can have a debilitating impact on their daily lives. The Secret Agent Society (SAS) program has been shown to be effective in improving the social skills of these children when delivered in a group setting. This pilot study evaluated whether individually delivered SAS would yield similar outcomes. Three participants were recruited for the 9-week intervention. Measures of social competence were administered at four points: pretest 1, pretest 2, post-intervention and at 6-weeks follow up. Participants showed significant improvement in half of the measures assessing social competence. On a third of these measures, two participants demonstrated improvements to within the range of their typically developing peers. Follow-up results suggested that improvements were maintained at 6-weeks post intervention. Limitations of this study and directions for future research are discussed. Key words: Autism, autism spectrum disorder, intervention, social skills.
\end{abstract}


An Evaluation of Individually Delivered Secret Agent Society Social Skills Program for Children with High-Functioning Autism Spectrum Disorders: A pilot study Social communication skills are paramount to forming meaningful social relationships (Owens, Granader, Humphrey, \& Baron-Cohen, 2008). Successful social interaction requires an array of skills including identifying and appropriately using non-verbal responses such as eye contact, gesture, facial expression, voice tone, interpersonal space, starting and maintaining conversations (Spence, 2003), as well as recognising emotions in others and adjusting responses accordingly (Barnhill \& Myles, 2001; McPartland \& Klin, 2006). While typically developing children acquire these skills usually without the need for direct teaching, individuals with Autism Spectrum Disorder (ASD) often find this a challenge. It is not uncommon for them to display inappropriate facial expressions, poor eye contact and/or respond with limited gestures (Woodbury-Smith \& Volkmar, 2009). In addition, children with ASD may experience difficulties engaging in reciprocal interactions, accepting suggestions from their peers and monitoring the effect their conversations and behaviours have on others (Attwood, 2000; Barry et al., 2003). Albeit unintentionally, they can also easily come across as insensitive due to their difficulties in offering comfort to others at times of stress or unhappiness (Klin \& Volkmar, 2003).

The impact of social skills impairment on children with ASD is extensive, especially among those with higher intellectual functioning (Klin, McPartland, \& Volkmar, 2005). These children are reported to have fewer friends than their typically developing peers (Koning \& Magill-Evans, 2001). They are stuck in a vicious cycle of social isolation - that is, they often desire close friendships with their peers, but fall short of the skills and experiences to form them (Nick Bauminger, 2002; NIck Bauminger \& Kasari, 2000). High-functioning children on the spectrum also 
demonstrate increased self-awareness of their social skills deficits (Knott, Dunlop, \& Mackay, 2006). This self-awareness, coupled with repeated social failures are reported to result in significantly higher levels of anxiety and depression among these children than their typically developing peers (Groden, Baron, \& Groden, 2006; Lopata et al., 2010). Their idiosyncratic behaviours can also easily make them targets of bullying and teasing (Little, 2001; Van-Roekel, Scholte, \& Didden, 2009).

Intervention programs aimed at teaching social skills have been introduced to attenuate the enduring impact of these social skills deficits (Cappadocia \& Weiss, 2011). Frankel and colleagues (2010) demonstrated the efficacy of a 12 -week Children's Friendship Training (CFT) social skills program for 76 high-functioning children aged 7-10 years with ASD. Target skills included conversational, play and negotiation skills. Post-intervention data revealed significant improvements on child measures of popularity, loneliness and parent measures of social skills. However, treatment gains did not generalise to the school setting, as indicated by teacher-report measures, and only parent-report improvements were maintained at 3-month followup. Cotugno (2009) evaluated the effectiveness of a 30-week social skills program for 18 children aged 7-11 years. Skill demonstrations, role-play exercises and ongoing feedback were provided to teach children the target skills of joint attention, anxiety and stress management, and coping with change. Although results at post-intervention demonstrated significant improvement across the targeted skills, the study was limited by the lack of a no-treatment ASD control group and the absence of follow-up data to assess the maintenance of treatment gains.

Despite the prevalence of social skills intervention for high-functioning children with ASD that demonstrate positive outcomes, investigations of the successful generalisation of skills outside treatment settings, the use of a non- 
treatment control group, and/or follow-up data remain scarce. Rao and colleagues (2008) reviewed ten social skills evaluation studies for high functioning individuals with ASD. While seven out of the ten reported positive treatment outcomes, only three studies had included components to promote skills generalisation. Nonetheless, only one study (Barry et al., 2003) demonstrated partial generalisation, while the other two (Barnhill, Cook, Tebbenkamp, \& Myles, 2002; Marriage, Gordon, \& Brand, 1995) reported disappointing results. Additionally, only one (Barry et al., 2003) out of the ten studies conducted follow-up assessment, albeit relatively short-term. Similar research gaps were also highlighted in recent review by Flynn and Healy (2012) where three studies (DeRosier, Swick, Davis, McMillen, \& Matthew, 2010; Dotson, Leaf, Sheldon, \& Sherman, 2010; Kroeger, Schultz, \& Newsom, 2007) reviewed demonstrated improvements in participants' social skills, but only one (Dotson et al., 2010) had collected generalisation and maintenance data. For this study, participants achieved only partial generalisation.

In view of this research gap, Beaumont and Sofronoff (2008) evaluated a 9session multi-component social skills program called the Secret Agent Society (SAS; formerly known as the Junior Detective Training Program) for high-functioning children with ASD aged 8-12. In this program, social skills learning is facilitated through a computer game, small group sessions, parent training sessions and teacher handouts. Results from the randomised controlled trial showed that participants who received the program had significant improvements in their social skills at home and in school, as indicated by parent- and teacher-report measures, with improvements maintained at 5-month follow-up. The program has since been made commercially available (see www.sst-institute.net for further details).

\section{The Current Study}


Originally designed as a small group-based intervention, SAS has also been tailored for individual delivery (Beaumont, 2010). This tailoring was undertaken because of the challenges some practitioners face in recruiting groups of children for the program, as well as Australia's publicly funded health insurance scheme only funding interventions delivered either individually or in groups of six or more people, thus limiting the public availability of SAS. This pilot study therefore aimed to extend Beaumont and Sofronoff's (2008) research and evaluate whether individually delivered SAS would result in similar improvements in children's social-emotional skills. With the program adopting a family-based approach in its delivery, the study included children's siblings and one or more caregivers, to examine the impact the intervention had on their social-emotional skills.

\section{Method}

\section{Participants}

Three boys were selected from 21 families who expressed interest in the project. Selection was based on meeting the inclusion criteria; that is, they were required to: (a) have a pediatrician diagnosis of Asperger's Disorder, High Functioning Autism or Pervasive Developmental Disorder—not otherwise specified according to the Diagnostic and Statistical Manual-Fourth Edition, Text Revision (DSM-IV-TR; American Psychiatric Association, 2000), (b) have a Wechsler Abbreviated Scale of Intelligence-Second Edition (WASI-II; Wechsler, 2011) prorated IQ score of 85 or above, (c) be aged 8-12 years at the time of intake assessment, (d) not be receiving any psychotherapy or social skills intervention during the course of study, and (e) have a typically developing sibling aged 8-12 years. Participants' parents were also required to have no more than mild symptoms of depression, anxiety and stress on the Depression, Anxiety, Stress Scales 21 (DASS 21; Lovibond 
\& Lovibond, 1995) and a Parenting Scale total score of less than 3.2 (Arnold, O'Leary, Wolff, \& Acker, 1993). This was to ensure that parents had the emotional and parenting capacity to support their children throughout the program.

Participant A was an 11-year-old boy with superior IQ who tended to engage in conversations with people on topics of his own interest. His parents noted that he was often not aware of his listeners' interest and would continue talking despite his audience disengaging. Appropriate eye contact was described as being limited except when he spoke about topics that he was interested in. At school, he had difficulty participating in group activities and maintaining conversations with his peers. Participant A also had a history of becoming easily upset over minor issues, but would calm down when hugged and listened to. His twin sister was not noted to have any significant developmental difficulties.

Participant B was an 11-year-old boy who enjoyed spending time with his schoolmates. Due to his "stickler for rules" character, however, he would easily become agitated when they misbehaved. His parents also noted that he had difficulty maintaining conversations, often leaving out important contextual information but expecting others to understand and then getting angry when they did not. Participant B was more often angry at home than when out of the house. He would become aggressive when angry and required a few hours to calm down. Diagnosed with attention deficit hyperactive disorder (ADHD) at the age of 3, he was prescribed methylphenidate to help him stay more focused at school. Participant B shared a close relationship with his 12-year-old brother.

Participant $\mathrm{C}$ was an 8-year-old boy who enjoyed playing with others and engaging in conversations of his own interest. He received speech pathology intervention when he was younger and his speech improved significantly. However, 
his parents reported that he was unable to initiate or join in conversations and activities with his peers. Turn-taking and extending conversations to topics outside of his areas of interest were described as difficult. He also had difficulty recognising emotions in himself and others. Participant $\mathrm{C}$ had a 21-year old brother and 10-year old sister whom he enjoyed playing with.

\section{Design}

The study staggered the introduction of the intervention by two weeks across participants and included a follow-up assessment 6-weeks after each participant completed the intervention.

\section{Measures}

Developmental History Questionnaire. Parents were asked to complete the questionnaire, adapted from Beaumont (2010) at the intake assessment to ensure that their child met the inclusion criteria for the study.

Childhood Asperger Syndrome Test (CAST; Scott, Baron-Cohen, Bolton, \& Brayne, 2002). The CAST was used to confirm that participants met DSM-IV-TR criteria for Asperger's syndrome. This test has high sensitivity and specificity (Williams et al., 2005), and has been shown to be effective in screening children with Asperger's syndrome in the general population (Scott et al., 2002). The clinical cut off score was 15 for possible Asperger's syndrome or related social communication difficulties.

\section{Wechsler Abbreviated Scale of Intelligence_-Second Edition (WASI-II;}

Wechsler, 2011). WASI-II, which has high internal consistency and acceptable to excellent concurrent validity with the Wechsler Intelligence Scale for Children-Fourth Edition (WISC-IV), was administered to the children at the intake assessment to assess their intellectual functioning and ensure they met the inclusion criteria. The 
four-subtest form, which comprises versions of the Similarities, Vocabulary, Block

Design and Matrix reasoning subtests of the WISC-IV, was used to generate an estimate of each child's full scale IQ (FSIQ).

Depression, Anxiety, Stress Scales 21 (DASS 21; Lovibond \& Lovibond, 1995). The DASS 21 is a 21 -item self-report measure that assesses depression, anxiety and stress in adults. The DASS 21 is widely used with high internal consistencies across the depression, anxiety and stress scales (Henry \& Crawford, 2005). The current study required parents to complete the DASS-21 to assess their emotional capacity to support their child through the program. To be eligible for the study, parents' were required to score within the non-clinical range for the DASS-21, that is, no more than mild symptoms of depression, anxiety and stress (Lovibond \& Lovibond, 1995).

The Parenting Scale (Arnold, O'Leary, Wolff, \& Acker, 1993). The 30item parenting scale was employed to assess the extent to which parents display dysfunctional parenting styles across three categories: overreactivity, laxness and hostility. Each item is presented on a 7-point Likert scale anchored with one effective and one ineffective strategy (e.g., When my child misbehaves: "I raise my voice or yell" versus "I speak to my child calmly"). Parents rate their tendency to use these strategies in response to their child's misbehaviour. High scores on the PS are reflective of dysfunctional parenting styles. This measure is reported to have adequate internal consistency across its scales (Rhoades \& O'Leary, 2007), and was used to confirm the capacity of parents to support their children through the program.

Social Skills Questionnaires (SSQ)—Parent (SSQ-P) and Teacher (SSQT; (Spence, 1995). The SSQ-P and SSQ-T are rating scales for parents and teachers to evaluate children's social competence. The scales require respondents to rate the 
applicability of each of 30 statements in relation to a child's behaviour over the past four weeks. Both SSQ-P and SSQ-T have good internal consistency (Butterworth et al., 2013), and have been used in previous studies (Beaumont \& Sofronoff, 2008; Mackay, Knott, \& Dunlop, 2007) to evaluate the efficacy of social skills interventions with children with AS. The current study used both SSQs to assess the social skills of the children, but only the SSQ-P was used for the siblings.

Emotion Regulation and Social Skills Questionnaire (ERSSQ)—Parent (ERSSQ-P) and Teacher (ERSSQ-T; Beaumont \& Sofronoff, 2008; Butterworth et al., 2013). The ERSSQ-P and ERSSQ-T, designed to evaluate the emotion regulation and social skills of high-functioning children on the spectrum, require respondents to indicate on a 5-point scale the frequency with which children exhibit various social-emotional behaviours. The ERSSQ-P consists of 27 items and the ERSSQ-T, 25 items. Both scales have shown high internal consistency (Butterworth et al., 2013) and were used in this study to evaluate participants' improvements in the specific social-emotional skills taught in the SAS intervention. The ERSSQ-P was also administered to assess siblings' social skills.

The Child and Adolescent Social Perception measure (CASP; MagillEvans, Koning, Cameron-Sadava, \& Manyk, 1995). The CASP was used in this study to assess children's abilities to infer the emotional state of others using contextual and non-verbal cues such as facial expressions and body postures. The measure contains 10 unrelated videotaped scenes portraying common day-to-day social interactions. The audio tracks to the videotaped scenes are filtered to make the sound incomprehensible, but leaving the tone and rate of speech as cues for the participants to describe the characters' emotional states as well as provide reasons for their answers. Recordings are standardised across children and individually 
administered. Two sets of scores, the Emotion Score (ES), which is based on correct identification of the characters' feelings and the Non Verbal Cues Score (NCS), which assesses the ability to accurately use the cues to infer emotions, were generated. Both ES and NCS have been shown to display good internal consistency (Koning, Magill-Evans, Volden, \& Dick, 2011). The measure has been previously used by researchers with AS populations (Castorina \& Negri, 2011; Koning et al., 2011).

James and the Maths Test (Attwood, 2004a). This task was used to assess participants' knowledge of anxiety management strategies. It involved the coinvestigator reading aloud to each participant a scenario about James, a boy who felt anxious about completing a math test in class. Participants were instructed to suggest strategies to help James cope with his anxiety. Administration was standardised across the participants, with the co-investigator transcribing responses and later awarding a point for each appropriate strategy. Scoring was checked for inter-rater reliability using an independent rater blind to the study. Inter-rater agreement was within acceptable range $(K=.81)$.

Dylan is Being Teased (Attwood, 2004b). This task, which assesses participants' knowledge of anger management strategies, involved a similar protocol to "James and the Maths Test" but using a different scenario. Participants were asked to provide suggestions for how the story character, Dylan, could better manage bullying at school. Scoring procedures mirrored that for "James and the Maths Test". Inter-rater agreement for the present study was within acceptable limits $(K=.91)$

Piers-Harris Self-Concept Scale (PHS; Piers, 1984). The PHS is an 80-item yes/no self-report measure that assesses how children and adolescents perceive themselves across 6 domains: physical appearance and attributes, intellectual and 
school status, happiness and satisfaction, behavioural adjustment, freedom from anxiety and popularity. This scale provides individual scores for each domain and a composite score that reflects a child's overall self-concept. In this study, the 12-item popularity subscale was used to evaluate participants' perceived popularity including their ability to make friends and involvement in social activities. The popularity subscale is internally consistent (Piers, 1984) and has been used previously to evaluate a social skills intervention for children with ASD (Frankel et al., 2010).

\section{Program satisfaction questionnaire-Parent and teacher forms}

(Beaumont, 2010). These questionnaires, specially designed to assess respondents' views on the appropriateness and effectiveness of the SAS program, were completed by parents and teachers following completion of the intervention.

\section{Procedure}

Recruitment. Participants were recruited via a number of disability support groups within the Perth Metropolitan area. Interested parents were provided the study information sheet and consent form. Upon receiving the parental consent, intake assessments consisting of the WASI-II, CAST, DASS-21 and parenting scale were conducted to assess children's eligibility for the study. Three participants and their siblings who met the eligibility criteria were recruited for the study.

Intervention. Pre-intervention assessments were administered to each participant prior to the commencement of the study (pretest 1) and the introduction of the intervention delivered at staggered intervals (pretest 2). To ensure that the parents were aware of the requirements of the SAS program, an initial parent information session was conducted. Intervention commenced the week following the briefing by the author who was trained to deliver the SAS program. The program of 9 weekly $75-$ minute sessions started with the author spending the first 60 minutes of each session 
engaging the child and his sibling in various SAS activities ${ }^{1}$. Each session ended with a 15 minutes discussion with the parents to review the skills taught. Weekly tip sheets were also provided to the teachers via the parents to ensure the skills learnt in the program were reinforced in school ${ }^{2}$. Post-intervention assessments were administered after the conclusion of the intervention.

Follow up. Follow-up assessments were administered at 6-weeks postintervention.

Treatment fidelity. To ensure treatment fidelity, the therapist completed session checklists indicating activities completed for each session. The overall mean percentage completion rate of session activities was $89 \%$, with a range between $36 \%$ and $100 \%$. All sessions were video-recorded and $20 \%$ of them were randomly checked by an independent observer to ensure accuracy of the checklist data. The reliability check found $96 \%$ agreement between raters.

Data Analysis. Clinical significance analysis based on Jacobson and Traux (1991) was conducted to investigate participants' pretest 2-post intervention and pretest 2-follow-up scores on each outcome variable. The analysis involved firstly assessing the statistical reliability of the change - that is, whether improvement in scores was greater than would be expected from measurement error. Change was defined as reliable when the reliable change index calculated as per formulae outlined by Jacobson and Traux (1991) was greater than 1.96. A test of clinical significance based on published normative data for the functional and clinical population was subsequently conducted to determine the clinical cut-off scores for each outcome measure. As per Jacobson and Truax's (1991) recommendations, where non-clinical

\footnotetext{
${ }^{1}$ With the exception of Participant $\mathrm{C}$ who received the final 2 sessions in a week.

2 With the exception of Participant $\mathrm{C}$ who underwent the final half of the program during the school holidays. In his case only the first three teacher's tip sheets were provided.
} 
norms were unavailable, a cut-off of two standard deviations above the clinical mean was used. Where clinical norms were unavailable, a cut-off of two standard deviations below the non-clinical mean was used. When both clinical and non-clinical norms were available, the cut-off would be the midpoint of the two aforementioned cut-offs. Clinically significant change was defined as: (a) a pretest 2 - score in clinical range, (b) a post-intervention and/or a follow-up score in the nonclinical range and (c) a reliable change score greater than 1.96 .

Further analyses were conducted to evaluate participants' overall performance across outcome measures. Two percentages were computed for each participant: (a) the percentage of measures with pretest 2 scores capable of showing reliable change that did show a reliable change, and (b) the percentage of measures with pretest 2 scores in the clinical range that evidenced a clinically significant change.

\section{Results}

Participants' scores on the outcome measures at pretest 1, pretest 2, postintervention and follow-up are presented in Table 1, and their overall performances across the outcome measures are presented in Table 2.

\section{Insert Table 1 and 2 about here}

Social skills and emotion regulation measures: Parent report. Participants' social and emotion regulation skills were assessed using the parent version of the SSQ and ERSSQ. Results at post-intervention indicated that all participants' SSQ-P and ERSSQ-P scores were within the non-clinical range. Specifically, on both measures, Participant B showed clinically significant improvement, Participant $\mathrm{C}$ demonstrated reliable improvement while Participant A remained unchanged. Siblings' scores on the SSQ-P and ERSSQ-P scores were generally unchanged and within non-clinical range, with the exception of Participant C's sister who showed reliable improvement 
on the SSQ-P. At follow-up, participant's scores were maintained. Participant C's sister's SSQ-P, while still within the non-clinical range, no longer met the reliable change criteria.

Social skills and emotion regulation measures: Teacher report. Analysis of teacher data was performed for two of the three participants whose teachers returned completed forms at pre- and post-intervention. Results on the SSQ-T indicated that Participant A showed reliable change and Participant B remained unchanged. At postintervention, both participants achieved SSQ-T scores within the non-clinical range. For the ERSSQ-T, both participants did not demonstrate a reliable change and scores remained within the clinical range. Follow up data were not available as the assessment coincided with the school holidays.

Emotion recognition. Changes in participants' emotion recognition skills were assessed using the CASP. Analysis of the CASP-ES's pre- and post-intervention scores revealed that only Participant A and B showed reliable improvement in successfully identifying the emotions of presented characters in the videos. However, these improvements were not clinically significant. At post-intervention, only Participant A reported CASP-ES scores within the non-clinical range. Follow up data revealed that while Participant A's and B's CASP-ES scores remained within the nonclinical and clinical range respectively, their scores had dropped such that they no longer met the criteria for reliable change. For the CASP-NCS, Participant A demonstrated clinically significant improvement at post-intervention, whereas Participant B's and C's responses suggested no change in these skills. At follow-up, Participant A maintained his performance on CASP-NCS.

Knowledge of emotion management strategies. Pre and post-intervention data from the Dylan is Being Teased measure revealed that Participant B showed clinically 
significant improvement in his knowledge of appropriate anger-management strategies. Participant $\mathrm{C}$ showed a reliable improvement but did not meet the criteria for clinical significance. Participant A's scores were unchanged and remained within the clinical range. At follow-up, Participant B maintained improvement while Participant $\mathrm{C}$ no longer met the reliable change criteria. For James and the Maths Test, none of the three participants recorded a reliable change in their pre-post knowledge of appropriate anxiety-management strategies. With the exception of Participant A, participants' scores on the James measure remained within the clinical range at post-intervention. At follow-up, Participant B met the criteria for clinical significance while Participant A and C remained unchanged.

Self-report popularity measure. Results from the PHS indicated that none of the three participants demonstrated reliable change in their self-reported popularity at post-intervention and/or at follow-up. Nonetheless, post-intervention and follow-up scores across participants were all within the normal range.

Program satisfaction questionnaire. Overall feedback of the individually delivered SAS program was positive. Exemplars of narrative feedback by parents and teachers are provided below:

The program equipped the children with the ability to calm themselves and regulate their emotions. He is much better at recognising emotions in himself and others now. We also have definite tools to point them to now, like the $\mathrm{O}_{2}$ regulator. (Participant C's mum)

He tends not to get as upset or angry. He listens to others' conversations and joins in. We are a little calmer. We have told him what behaviours we expect of him and reinforce them positively. (Participant B's mum) 
His emotional development has been so important in the way he reacts to his peers and has been delightful to watch. It is great to see his social \& emotional growth. I think the program has been very worthwhile for him. (Participant A's teacher)

\section{Discussion}

Two participants, Participant B and C, showed reliable change on the parentreport social skills and emotion regulation measures. Participant B, in particular, achieved clinical significance on both measures. Participant A's performance on these measures did not meet criteria for either reliable or clinically significant change; this was likely due to a ceiling effect on these measures that rendered it difficult for improvement to be demonstrated. Participant A's post-intervention scores on both the parent-report measures were higher than Participant B who met the criteria for clinical significance on the same measures. Furthermore, all participants were scoring within the non-clinical range at post intervention on the parent-report measures. As predicted, participants' performance on the parent-report measures of social skills and emotion regulation were maintained at 6-weeks follow up. This suggests that treatment gains were generally enduring, although a longer-term follow-up study would be required to verify this.

Teacher-reports of participants' social skills and emotion regulation were mixed. Participant A demonstrated reliable change on the SSQ-T while Participant B's performance remained unchanged within the non-clinical range. The finding that Participant A had improved on the SSQ-T is particularly encouraging given that the SSQ was designed to assess important positive social interactions among typically developing children. Although it may be expected that this improvement would also be reflected on the ERSSQ-T, reliable change was not demonstrated on Participant 
A's ERSSQ-T. This may be partially due to the limited sensitivity of the SSQ to capture the subtle social difficulties often experienced by children with ASD relative to the ERSSQ-T, which was specifically developed to assess the social-emotion challenges that characterise children on the spectrum (Beaumont \& Sofronoff, 2008; Butterworth et al., 2013). Nonetheless, Participant A's pre-post ERSSQ-T scores were trending towards a reliable change and teacher feedback on the program satisfaction questionnaire highlighted his social and emotional growth (i.e., "Participant A has become more interactive with his peers and has been able to deal with mistakes he makes better than previously"). At post-intervention, Participant B's scores on both SSQ-T and ERSSQ-T had decreased, albeit the change was not considered reliable. It should be noted that parents and teacher of Participant B reported an extremely stressful period for him nearing the end of the intervention phase, having classmates who were exceptionally disruptive and misbehaving at school. Caution must therefore be exercised when interpreting these scores, as external factors are likely to influence one's ability to interact and regulate their emotions in social situations. As such, no conclusive interpretations can be drawn about the generalisation of participants' social-emotional skills to the school environment. Follow-up data from teachers was also unavailable as the assessment coincided with the school holidays.

Despite having had high scores at pretest, one sibling continued to demonstrate reliable change on the SSQ. At follow-up, the sibling's score was no longer reliably greater than at pre-treatment (although it remained near perfect). As for the other two siblings, near perfect scores reported at pre-intervention may have significantly reduced their potential to show a reliable improvement.

Participant A demonstrated reliable change on the CASP-ES and clinically significant improvement on the CASP-NCS. The latter was maintained at 6-weeks 
follow-up, but his CASP-ES scores had slipped, albeit remaining within the nonclinical range. This suggests that Participant A's ability to attend to non-verbal cues in emotion recognition was within the performance of his typically developing peers by the conclusion of the intervention and at follow-up. While Participant B's scores on the CASP-NCS were unchanged, reliable pre- to post-intervention improvement was noted on the CASP-ES, although this was not maintained at follow-up. Caution is warranted when interpreting these findings, as Participant B was avoidant of the CASP task after having completed it several times over the course of the study. Arguably, this lack of motivation may have impaired his CASP performance. There was no reliable change in Participant C's CASP post-intervention and follow-up scores relative to pre-intervention. This is likely due to the discrepancy between the emotions focused in the intervention with participant $\mathrm{C}$; that is, more emphasis on simple as opposed to complex emotions, and those assessed in the CASP; that is, more complex emotions such as those with incongruent situational and emotional cues. Alternate emotion recognition measures such as The Cambridge Mindreading Face-Voice Battery for Children (Golan \& Baron-Cohen, submitted), which assess both basic and complex emotions, are recommended for future studies. Nevertheless, feedback provided by his parents on the program satisfaction questionnaire indicated that Participant $\mathrm{C}$ had developed better emotion-recognition skills, although the change was not captured on the CASP.

On measures of knowledge of emotion management strategies, postintervention results indicated that Participant $\mathrm{B}$ and $\mathrm{C}$ demonstrated reliable improvement on the Dylan but not on the James measure. Specifically, Participant B's performance on the Dylan measure improved from the clinical to the normal range. The finding that both participants demonstrated improvements in their knowledge of 
appropriate anger but not anxiety management strategies was particularly interesting. This may be attributed to the fact that Participant B and C each identified themselves as angry rather than anxious children. As such, they may have had more opportunities to review and practice the anger-management (as opposed to anxiety-management) strategies taught in the program in everyday contexts, resulting in higher scores on the anger-management as opposed to the anxiety-management measure. At follow-up, Participant B maintained improvement on the Dylan measure while Participant C no longer met criteria for reliable change. However, it should be noted that Participant C received most of the intervention during the school holidays. In the absence of opportunities at school for skills rehearsal and application, it is not surprising that Participant $\mathrm{C}$ did not maintain treatment gains at follow-up. Importantly, on the James measure, Participant B showed clinically significant improvement at follow-up. This is consistent with results on the Dylan measure and parent feedback on the program satisfaction questionnaire, which noted improvements in his knowledge of emotions regulation strategies and ability to stay calm. For Participant A, performance on the James and Dylan measures was unchanged throughout the study. It is possible that this was due to him having high scores on both measures at pre-test relative to Participants B and C, and hence having less room to show reliable improvement.

Post-test and follow-up scores of self-reported popularity for all participants remained unchanged. While previous studies have reported that children with ASD have a lower self-concept (Capps, Sigman, \& Yirmiya, 1995), the observed high scores at pre-intervention, post-intervention and at follow-up instead suggest that participants in the current study either had high quality friendships and positive selfidentity (Nirit Bauminger, Shulman, \& Agam, 2004), or a lack of awareness of their social and/or friendship challenges. 


\section{Limitation and Future Directions}

Several methodological limitations of the current study must be acknowledged. Firstly, as with studies with small $\mathrm{N}$ designs and only male participants, replication with a larger sample and female participants is warranted to ensure that current results can be generalised to a larger population. Secondly, verbal reports and participants' behaviour suggested that repeat administration of measures at pretest 1 , pretest 2 , post-intervention and at follow-up affected participants' motivation to complete the tasks. Since motivation is essential to ensure optimal performance, it is recommended that future studies limit test administration to a preand post-assessment. Thirdly, medication use, as a confounding factor, was not monitored throughout the course of this study. This limitation should be addressed in future evaluation trials to ensure participants maintain a stable dose of medication use during the study. In addition, it must be acknowledged that changes were made to the protocol for one participant (i.e., sessions being collapsed across time and conducted over the holidays) and data was also not collected on whether teachers received the weekly tip sheets from parents. Although these factors reflect "real-world" practice, they may have limited the potential impact of the intervention.

Future research should also address other limitations of this study, specifically with the delivery and choice of measures used. This includes the reliance on parent and teachers report whose responses may have been positively biased by expectations of improvements, given that they were not blind to the study. The inclusion of behavioural observations as an unbiased measure would allow for more rigorous evaluation of treatment outcome. Although the current study had screened participants' diagnoses using the CAST, the use of gold standard screening tool such as the ADOS and ADI-R would have been much preferred. This was not possible due 
to the cost and limited availability of training in such measures. Additionally, it is recommended for the entirety of the intervention to be conducted within the school term, not only to allow for more accurate assessment of participants' own self-report popularity among peers, but also enable follow-up assessments with the teachers. The absence of self-report emotion regulation in the current study should also be acknowledged as a limitation. This did not occur as research has shown that limited self-awareness among higher-functioning individuals with ASD (Mazefsky, Kao, \& Oswald, 2011) could compromise the test validity of self-report measures. Despite these limitations, the use of multi-method approach to assessing participants' social skills and emotional regulation highlights the rigorous evaluation adopted by the current study.

\section{Conclusion and Clinical Implications}

Notwithstanding the limitations highlighted above, this exploratory study provided preliminary support for the effectiveness of the SAS program delivered individually. All three participants who completed the individually delivered SAS program showed significant improvements on half of the measures assessing socialemotional competence. Two of the participants demonstrated improvements to within the range of their typically developing peers on a third of these measures. Follow-up results suggested that improvements were maintained at the 6-week post intervention. This is an important finding for both professionals and families given there have previously been no formal evaluations of the program delivered in this format. Finally, with the practical ease of delivering services individually versus in a group format, the current findings are an important first step in extending the reach of the program to families with an ASD child who requires social skills training. 


\section{Reference List}

Arnold, D., O'Leary, S., Wolff, L., \& Acker, M. (1993). The parenting scale: A measure of dysfunctional parenting in discipline situations. Psychological Asssment, 5, 137-144.

Attwood, T. (2000). Strategies for improving the social integration of children with Asperger Syndrome. Autism, 4, 85-100.

Attwood, T. (2004a). James and the maths test. In Exploring feelings: Cognitive behaviour therapy to manage anxiety. Arlington, TX: Future Horizons Inc.

Attwood, T.. (2004b). Dylan is being teased. In Exploring feelings: Cognitive behaviour therapy to manage anger. Arlington, TX: Future Horizons.

Barnhill, G., Cook, K., Tebbenkamp, K., \& Myles, B. (2002). The effectiveness of social skills intervention targetting nonverbal communication for adolescents with asperger syndrome and related pervasive developmental delays. Focus on Autism and other developmental disabilities, 17, 112-118.

Barnhill, G., \& Myles, B. (2001). Attributional style and depression in adolescents with Asperger syndrome. Journal of Positive Behavior Interventions, 3, 175183.

Barry, T. D., Klinger, L., Grofer, L., Joycelyn M., Palardy, N., Gilmore, T., \& Bodin, S. D. (2003). Examining the effectiveness of an outpatient clinic-based social skills group for high-functioning children with autism. Journal of Autism and Developmental Disorders, 33, 685-701. doi:0162-3257/03/1200-0685/0

Bauminger, N. (2002). The facilitation of social-emotional understanding and social interaction in high-functioning children with autism: Intervention outcomes. Journal of Autism and Developmental Disorders, 32, 283-298. doi:0162$3257 / 02 / 0800-0283 / 0$ 
Bauminger, N., \& Kasari, C. (2000). Loneliness and friendship in high-functioning children with autism. Child Development, 71, 447-456. doi:0009$3920 / 2000 / 7102-0014$

Bauminger, N., Shulman, C., \& Agam, G. (2004). The link between perceptions of self and of social relationships in high-functioning children with autism. Journal of developmental and physical disabilities, 16, 193-214. doi:1056$263 X / 04 / 0600-0193 / 0$

Beaumont, R. (2010). The SAS Facilitator Manual. Queensland, Australia: The Social Skills Training Institute.

Beaumont, R., \& Sofronoff, K. (2008). A multi-component social skills intervention for children with Asperger syndrom: The Junior Detective Training Program. Journal of Child Psychology and Psychiatry, 49, 743-753. doi:10.1111/j.14697610.2008.01920.x

Butterworth, T., Hodge, M. A. R., Sofronoff, K., Beaumont, R., Gray, K. M, Roberts, J., . . Einfield, S. L. (2014). Validation of the Emotion Regulation and Social Skills Questionnaire for young people with Autism Spectrum Disorders. Journal of Autism and Developmental Disorders, 44, 1535-1545. doi:10.1007/s10803-013-2014-5

Cappadocia, M. C., \& Weiss, J. A. (2011). Review of social skills training groups for youth with Asperger syndrom and high functioning Autism. Research in Autism Spectrum Disorders, 5, 70-78.

Capps, L., Sigman, M., \& Yirmiya, N. (1995). Self-competene and emotional understanding in high-functioning children with autism. Development and Psychopathology, 7, 137-149. doi:10.1017/S0954579400006386 
Castorina, L. L., \& Negri, L. M. (2011). The inclusion of siblings in social skills training groups for boys with Asperger Syndrome. Journal of Autism and Developmental Disorders, 41, 73-81.

Cotugno, A. (2009). Social competence and social skills training and intervention for children with autism spectrum disorders. Journal of Autism and Developmental Disorders, 39, 1268-1277. doi:10.1007/s10803-009-0741-4

DeRosier, M. E., Swick, D. C., Davis, N. O., McMillen, J. S., \& Matthew, R. (2010). The efficacy of a social skills group intervention for improvement social behaviors in chilren with high-functioning autism specturm disorders. Journal of Autism and Developmental Disorders, 1402-1403. doi:10.1007/s10803-010$1128-2$.

Dotson, W. H., Leaf, J. B., Sheldon, J. B., \& Sherman, J. A. (2010). Group teaching of conversational skills to adolesents on the autism specturm. Research in Autism Spectrum Disorders, 4, 199-209. doi:10.1016/j.rasd.2009.09.005.

Flynn, L., \& Healy, O. (2012). A review of treatments for deficits in social skills and self-help skills in autism spectrum disorder. Research in Autism Spectrum Disorders, 6, 431-441. doi:10.1016/j.rasd.2011.06.016

Frankel, F., Myatt, R., Sugar, C., Whitham, C., Gorospe, C. M., \& Laugeson, E. (2010). A randomized controlled study of parent-assisted children's friendship training with children having autism spectrum disorders. Journal of Autism and Developmental Disorders, 40, 827-842. doi:10.1007/s10803-009-0932-z

Golan, O., \& Baron-Cohen, S. (2006). The Cambridge Mindreading Face-Voice Battery for Children (CAM-C): Testing basic and complex emotion recognition in children with and without autism spectrum conditions. Unpublished manuscript: University of Cambridge. 
Groden, J., Baron, M. G., \& Groden, G. (2006). Assessment and coping strategies. In. In M. G. Baron, J. Groden, G. Groden \& L. P. Lipsitt (Eds.), Stress and coping in autism (pp. 15-41). New York, NY: Oxford University Press.

Henry, J., \& Crawford, J. (2005). The short-form version of the Depression Anxiety Stress Scales (DASS-21): Construct validity and normative data in a large non-clinical sample. British Journal of Clinical Psychology, 44, 227-239. doi:10.1348/014466505X29657

Klin, A., McPartland, J., \& Volkmar, F. (2005). Asperger syndrome. In. In F. Volkmar, R. Paul, A. Klin \& D. Cohen (Eds.), Handbook of Autism and Pervasive Developmental Disorders: Vol 1. Diagnosis, Development, Neurobiology, and Behaviour (3 ed., pp. 88-125). Hoboken, NJ: Wiley.

Klin, A., \& Volkmar, F. R. (2003). Asperger syndrome: Diagnosis and external validity. Child and Adolescent Psychiatric Clinics in North America, 12, 1-13.

Knott, F., Dunlop, A.-W., \& Mackay, T. (2006). Living with ASD: How do children and their parents assess their difficulties with social interaction and understanding. Autism, 10, 609-617. doi:10.1177/1362361306068510

Koning, C., \& Magill-Evans, J. (2001). Social and language skills to adolescent boys with Asperger syndrome. Autism, 5, 23-36. doi:10.1177/1362361301005001003

Kroeger, K. A., Schultz, J. R., \& Newsom, C. (2007). A comparison of two groupdelivered social skills programs for young children with autism. Journal of Autism and Developmental Disorders, 37, 808-817. doi:10.1007/s10803-0060207-x. 
Little, L. (2001). Peer victimisation of children with Asperger spectrum disorders. Journal of the American Academy of Child \& Adolescent Psychiatry, 40, 995996.

Lopata, C., Toomey, J., Fox, J., Volker, M. A., Chow, S., Thomeer, M. L., . . . Shmerbeck, A. (2010). Anxiety and depression in children with HFASDs: Symptom levels and source differences. Journal of Abnormal Child Psychology, 38, 765-776.

Lovibond, S. H., \& Lovibond, P. F. (1995). Manual for the Depression Anxiety Stress Scales. Sydney, Australia: The Psychology Foundation of Australia Inc.

Mackay, T., Knott, F., \& Dunlop, A.-W. (2007). Developing social interaction and understanding in individuals with Autism Spectrum Disorder: A groupwork intervention. Journal of Intellectual \& Developmental Disability, 32, 279-290. doi:10.1080/13668250701689280

Magill-Evans, J., Koning, C., Cameron-Sadava, A., \& Manyk, K. (1995). The child and adolescent social perception measure. Journal of Nonverbal Behavior, 19, 151-169.

Marriage, K. J., Gordon, V., \& Brand, L. (1995). A social skills group for boys with Asperger's. Australia and New Zealand Journal of Psychiatry, 29, 58-62.

Mazefsky, C. A., Kao, J, \& Oswald, D. P. (2011). Preliminary evidence suggesting caution in the use of psychiatric self-report measures with adolescents with high-functioning autism spectrum disorders. Research in Autism Spectrum Disorders, 5, 164-174. doi:10.1016/j.rasd.2010.03.006

McPartland, J., \& Klin, A. (2006). Asperger's syndrome. Adolescent Medicine Clinics, 17, 771-788. 
Owens, G., Granader, Y., Humphrey, A., \& Baron-Cohen, S. (2008). Lego therapy and the social use of language programme: An evaluation of two social skills interventions for children with high-functioning Autism and Asperger Syndrome. Journal of Autism Developmental Disorder, 38, 1944-1957. doi:10.1007/s10803-008-0590-6

Piers, E. V. (1984). Piers-Harris children's self concept scale-revised manual. Los Angeles, CA: Western Psychological Services.

Rao, P. A, Beidel, D. C., \& Murray, M. J. (2008). Social skills intervention for children with Asperger's syndrome or High-functioning Autism: A review and recommendations. Journal of Autism and Developmental Disorders, 38, 353361. doi:10.1007/s10803-007-0402-4

Rhoades, K. A, \& O'Leary, S. G. (2007). Factor structure and validity of the parenting scale. Journal of Clinical Child and Adolescent Psychology, 35, 137-146.

Scott, F. J., Baron-Cohen, S., Bolton, P;, \& Brayne, C. (2002). The CAST (Childhood Asperger Syndrom Test): Preliminary development of a UK screen for mainstream primary-school-age children. Sage Publication and The National Autistic Society, 6, 9-31. doi:10.1177/1362361302006001003

Spence, S. H. (1995). Social skills questionaire. In Social skills training: Enhancing social competence with children and adolescents: Photocopiable resource book. Winsor, England: NFER-Nelson.

Spence, S. H. (2003). Social skills training with children and young people: Theory, evidence and practice. Child and Adolescent Mental Health, 8, 84-96.

Van-Roekel, E., Scholte, R. H., \& Didden, R. (2009). Bullying among adolescents with autism spectrum disorders: Prevalence and perception. Journal of Autism and Developmental Disorders, 40, 63-73. doi:10.1007/s10803-009-0832-2 
Wechsler, D. (2011). Wechsler Abbreviated Scale of Intelligence-Second Edition (WASI-II). San Antonio, TX: NCS Pearson.

Williams, J., Scott, F., Stott, C., Allison, C., Bolton, P., Baron-Cohen, S., \& Brayne, C. (2005). The CAST (Childhood Asperger Syndrome Test). Sage Publication and The National Autistic Society, 9, 45-68. doi:10.1177/1362361305049029

Woodbury-Smith, M. R, \& Volkmar, F. R. (2009). Asperger syndrome. European Journal of Adolescent Psychiatry, 18, 2-11. doi:10.1007/s00787-008-0701-0 
Table 1

Psychometric Measures: Overall Results and Outcomes

\begin{tabular}{|c|c|c|c|c|c|c|c|c|c|c|c|c|}
\hline \multirow[t]{2}{*}{ Measures } & \multicolumn{4}{|c|}{ Participant A } & \multicolumn{4}{|c|}{ Participant B } & \multicolumn{4}{|c|}{ Participant C } \\
\hline & T1 & $\mathrm{T} 2$ & T3 & T4 & T1 & $\mathrm{T} 2$ & T3 & T4 & T1 & $\mathrm{T} 2$ & T3 & T4 \\
\hline SSQ-P & 51 & 54 & 54 & 56 & 22 & 24 & $48^{\mathbf{4}}$ & $47^{\mathbf{4}}$ & 35 & 40 & $57^{\Delta}$ & $49^{\Delta}$ \\
\hline SSQ-T & 43 & 35 & $45^{\Delta}$ & - & 50 & 49 & 46 & - & 55 & 38 & - & - \\
\hline ERSSQ-P & 67 & 76 & 79 & 80 & 31 & 36 & $65^{\star}$ & $74^{4}$ & 50 & 61 & $82^{\Delta}$ & $74^{\Delta}$ \\
\hline ERSSQ-T & 62 & 59 & 69 & - & 57 & 66 & 60 & - & 63 & 55 & - & - \\
\hline CASP-ES & 20 & 26 & $38^{\Delta}$ & 35 & 17 & 14 & $24^{\Delta}$ & 12 & 25 & 21 & 23 & 21 \\
\hline CASP-NCS & 31 & 23 & $51^{\wedge}$ & $46^{\star}$ & 18 & 18 & 21 & 19 & 14 & 10 & 17 & 13 \\
\hline PHS & 7 & 9 & 10 & 11 & 10 & 10 & 11 & 10 & 12 & 10 & 11 & 10 \\
\hline Dylan is being teased & 5 & 5 & 4 & 8 & 2 & 1 & $7^{\mathbf{4}}$ & $7^{\mathbf{4}}$ & 2 & 1 & $6^{\Delta}$ & 1 \\
\hline James and the maths test & 4 & 4 & 5 & 5 & 1 & 1 & 2 & $4^{\mathbf{4}}$ & 1 & 1 & 3 & 1 \\
\hline SSQ- P (Sibling) & 60 & 60 & 60 & 60 & 59 & 59 & 60 & 60 & 57 & 51 & $60^{\Delta}$ & 58 \\
\hline ERSSQ-P (Sibling) & 108 & 108 & 108 & 108 & 102 & 105 & 97 & 105 & 85 & 80 & 80 & 77 \\
\hline
\end{tabular}

Note. $\mathrm{T} 1$ = Pretest 1; T2 = Pretest 2; T3 = Post-intervention; T4 = 6-weeks follow-up; SSQ-P = Social Skills Questionnaire Parent form; SSQ-T = Social Skills Questionnaire-Teacher form; ERSSQ-P = Emotion Regulation and Social Skills Questionnaire-Parent form; ERSSQ-T = Emotion Regulation and Social Skills Questionnaire-Teacher form; CASP-ES = Child and Adolescent Social Perception Measure- Emotion Score; CASP-NCS = Child and Adolescent Social Perception Measure-Non Verbal Cue Score; PHS = Piers-Harris Self Concept Scale; ${ }^{\Delta}$ Statistically reliable change; ${ }^{\wedge}$ Statistically reliable and clinically significant improvement. 
INDIVIDUALLY DELIVERED SECRET AGENT SOCIETY

Table 2

Percentage of Measures that Evidenced a Reliable Change and Clinically $S$ Change

\begin{tabular}{ccc}
\hline Participant & Reliable change & Clinically Significant C \\
\hline A & $43 \%(3 / 7)$ & $33 \%(1 / 3)$ \\
B & $50 \%(4 / 8)$ & $43 \%(3 / 7)$ \\
C & $50 \%(3 / 6)$ & $0 \%(0 / 3)$
\end{tabular}

Note. Number of measures that showed reliable change and clinically signif change out of those possible in parentheses. 